\title{
RESECCIÓN TRANSURETRAL EN CÁNCER DE PRÓSTATA, UN PROCEDIMIENTO RADICAL. EXPERIENCIA CON 1017 CASOS
}

\author{
M. A. Reuter, M. Corredera², W. Epple, G. Ungemach, M. L. Verger y K. Dietz'.
}

Departamento de Urología. KOK Hospital Stuttgart. Alemania.

'Departmento de Biometría Medical. Univ. Tübingen. Alemania.

${ }^{2}$ Departamento de Urología. La Palma. Canarias. España.

Resumen.- OBJETIVO: Desde el comienzo de la resección transuretral RTU en 1931 el reseccionista siempre tuvo en mente resecar el cáncer de próstata. Sin embargo, su práctica no tuvo éxito por deficiencias instrumentales y técnicas. La primera resección transuretral por cáncer de próstata RTUPC se llevó a cabo en nuestra Institución en 1957 por Hans J. Reuter. La irrigación a baja presión permitió una resección segura de la próstata incluyendo la cápsula. De esta forma iniciamos en 1985 un estudio prospectivo para verificar si la RTUPC era un procedimiento radical.

MÉTODO: La RTUPC requiere una irrigación continua a baja presión con el reservorio de líquido anclado a la mesa de operaciones. El nivel de líquido debe mantenerse por debajo de 20 centímetros respecto a la región púbica en posición de litotomía. El flujo continuo con un trocar suprapúbico y un resectoscopio con vaina de calibre 28 Ch. Es indispensable una unidad de electro-bisturí autorregulada para adecuar la corriente de alta frecuencia a la resistencia al corte de los tejidos a resecar para que este sea preciso. El video monitoreo es obligado. La próstata se reseca completamente con su cápsula y algo de la grasa periprostática así como las vesículas seminales. Las muestras de tejido son etiquetadas por grupos para garantizar un correcto estadiaje histopatológico. Si la linfadenectomía laparoscópica está indicada, esta se llevará a cabo. A las 12 semanas se practica una segunda sesión de control de márgenes positivos.

RESULTADOS: Desde 1985 al 2004, 1017 pacientes con una media de edad de 68,9 años y cáncer de próstata localizado fueron resecados por 5 cirvianos diferentes con intención curativa. El porcentaje de estadios cancerosos fue de T1: 12, T2: 43, T3: 41, T4: 4. El grado de G1: 8, G2: 72, G3:20. La mortalidad fue de 1 sobre 1017. Precisaron de incisión de cuello vesical el 8,9\%. Incontinencia grado 3: Ninguno.

La supervivencia postoperatoria a los 10 años fue del $82 \%$ en los T1, 87\% en los T2, $81 \%$ en los T3 y del $34 \%$ en los T4. La recidiva bioquímica definida como el aumento de tres valores consecutivos del PSA sin limite inferior, fue en 5 años del 15\% en los T1, 19\% en los T2 y $27 \%$ en los T3.

CONCLUSIONES: Considerando nuestros resultados, concluimos que la Resección Transuretral del Cáncer de Próstata (RTUPC) es tan radical como la cirugía abierta. Los resultados de supervivencia y la recidiva del PSA son comparables, la tasa de incontinencia es mas baja que con la cirugía abierta. Es obligatoria la irrigación a baja presión utilizando el trocar suprapúbico, vaina del 28 Ch. y un electrobisturí autorregulado, la videomonitorización y un reseccionista con experiencia. 
Palabras clave: RTU. Cáncer prostático. Procedimiento radical. Supervivencia. Recidiva bioquímica

Summary.- OBJECTIVES: From the beginning of TUR in 1931 the reseccionists thought of resecting prostate cancer. Execution however failed for deficient instruments and techniques. The first transurethral resection for prostate cancer: TURPC - was performed at our institution in 1957 by Hans J. Reuter. Low pressure irrigation enabled safe transurethral resection of the prostate including the capsule. Thus we started in 1985 a prospective study to verify TURPC as a radical procedure.

METHOD: TURPC requires continuous low pressure irrigation with irrigating reservoir fixed at the operating table. The liquid level within is to maintain less than $20 \mathrm{~cm}$. water above the pubic region in lithotomy position. Continuous flow is maintained by suprapubic trocar and a resectoscope with 28Fr. sheath. An autoregulated electro-surgical unit is indispensable. It automatically adjusts the high-frequency current to suit the cut tissue's electrical resistance for precise cutting. Videomonitoring is mandatory. The prostate is resected completely with its capsule into periprostatic fat together with the seminal vesicles. The specimen is retrieved in fractions to guarantee correct histopathological staging. If indicated laparoscopic staging lymphadenectomy is performed. A secondary session for control of positive margins follows after 12 weeks.

RESULTS: From 1985 - 20041017 patients with a mean age of 68.9 years and with clinically localized prostate cancer were resected by 5 surgeons with curative intention. The cancer stage distribution was in \%:

T1: 12, T2: 43, T3: 41, T4: 4. - G1: 8, G2: 72, G3: 20. Mortality 1 out of 1017. Bladder neck incision 8.9\%. Incontinence grade 3: none. Postoperative survival over 10 years was $82 \%$ in $T 1,87 \%$ in $12,81 \%$ in T3 and 34\% in T4 patients. Biochemical recurrence as of the rise of three consecutive PSA-values was within 5 years $15 \%$ in $\mathrm{Tl}, 19 \%$ in $\mathrm{T} 2,27 \%$ in $\mathrm{T} 3$.

CONCLUSIONS: Considering our results we conclude that prostate cancer can be resected transurethrally as radical as with open surgery. The outcome as to survival and PSA-recurrence is comparable, the incontinence rate is less then with open surgery. It is mandatory to have low pressure irrigation with suprapubic trocar, $28 \mathrm{Fr}$. sheath and an autoregulated electrosurgical unit, video monito-ring and a well experienced reseccionist.

Keywords: TURP. Prostate cancer. Radical procedure. Postoperative survival. PSA-recurrence.

\section{INTRODUCCIÓN}

Desde los comienzos de la RTU en 1931, los reseccionistas siempre han pensado en resecar el cáncer de próstata. Sin embargo, el tratamiento siempre fue incompleto y problemático. La fase inicial de la RTUPC comenzó cuando, en 1957 realizamos la primera RTUPC en nuestro departamento de Stuttgart. En 1980 Hans J. Reuter publicó 323 casos en su Atlas de Endourología (1). Impulsados por esta idea, urólogos de todo el mundo han llevado a cabo este procedimiento, sin éxito sólo por no tener en cuenta un pequeño detalle del mismo. Este hecho, extremadamente importante, es la irrigación a baja presión. La consecuencia que se obtuvo fue extravasación de líquido. Frente a esto, uno de los usuarios más constantes de la irrigación a baja presión prosiguió con el procedimiento de la RTUPC de forma exitosa: Erich Matouschek en Karlsruhe en 1987 publicó las indicaciones de la técnica (2). Naturalmente se fue adaptando esta al estándar oncológico y se dió comienzo a un estudio prospectivo en Stuttgart en 1986 (5). Este artículo es la revisión de 19 años de experiencia y mejoras de la RTUPC.

El "estándar de oro" actual es la cirugía abierta, con prostato-vesiculectomía con exéresis completa de la glándula prostática incluyendo el verumontanum y las vesículas seminales, y, si está indicada, linfadenectomía de estadiaje. Similar a la cirugía, con la RTUPC a baja presión, se alcanza una exéresis completa del tumor, de la cápsula prostática y de las vesículas seminales. Esto se complementa con linfadenectomía laparoscópica de estadiaje.

\section{INDICACIONES}

Las indicaciones de la RTUPC incluyen a aquellos pacientes con cáncer de próstata localizado comprobado histológicamente, estadios T1-2 y T3a determinados con estadiaje preoperatorio mediante Examen Dígito Rectal (EDR), ecografía transrectal, Antígeno Prostático Específico (PSA), gammagrafía ósea, Tomografía Computarizada (TAC-scan) y radiología pulmonar. La enfermedad metastásica excluye al paciente del estudio. Se incluyen aquellos pacientes que no son subsidiarios o que rechazan la cirugía abierta. Los pacientes con un ASA 3 de alto riesgo (American Society of Anesthesiologists) también se incluyen, así como aquellos con recurrencia bioquímica de PSA tras radioterapia o braquiterapia y aquellos con condicionamientos religiosos, p. ej. testigos de Jehová. Ni la edad ni la obesidad limitaron las indicaciones para ser intervenidos. 


\section{MATERIAL}

Los pacientes diagnosticados de cáncer de próstata localizado comprobado fueron intervenidos entre enero de 1985 y diciembre del 2004. Fueron incluidos un total de 1017 casos, con una edad media de 69 años y un rango de edad de 40-89 años. Quinientos setenta y ocho (57\%) tenían 70 años o menos en el momento de la intervención. El tiempo medio de RTU fue de 60 minutos $(n=310)$ y la media de tejido resecado fue de 32 gramos (mínimo 4 , máximo $200 ; n=840$ ). La solución anestésica fué del $46 \%$ para ASA-1, $51 \%$ para ASA-2 y $3 \%$ para ASA-3 ( $\mathrm{n}=302$ ). La RTUPC la practicaron 5 cirujanos diferentes $(n=894)$.

La recogida de datos se extiende a través de un largo periodo, puesto que comprende continuas mejoras del procedimiento quirúrgico. Los dos cambios más importantes fueron la sustitución de la linfadenectomía abierta por la laparoscópica y la incorporación de la unidad electro quirúrgica de corriente autorregulada (Tabla I).

\section{MÉTODO}

La condición previa más importante para lograr un buen resultado con la RTUPC es la irrigación a baja presión con el trocar suprapúbico $(3,7)$. El reservorio de irrigación debe ser instala- do en la mesa operatoria. El nivel del líquido de irrigación no debe exceder de una altura de 10 ó, como máximo, 20 centímetros sobre la región púbica del paciente en posición de litotomía ( $\mathrm{Fi}$ gura 1). Su efecto es el que la vejiga permanezca prácticamente vacía a lo largo de la intervención, consiguiendo que la baja presión estática sea cercana a la intraabdominal $(4,5)$. Esto se logra fijando una bolsa de orina de 2500 c.c. instalada simplemente en posición en la mesa operatoria. La bolsa se mantendrá fláccida con un nivel de llenado de entre 1000-1500 c.c. El tubo desde el reservorio principal se conecta a la entrada de flujo de la bolsa (postoperatoriamente se conectará a la sonda transuretral) y la salida valvular del fondo de la bolsa se conecta a medida con un tubo ancho a la vaina del resectoscopio.

La solución irrigadora se fabrica con una concentración semiosmolar de manitol/sorbitol no electrolítica.

La vaina del resectoscopio debe ser del 27 28 Ch. con la punta clásica biselada (pico de pato) (Figura 2). Estas medidas dan lugar a un flujo laminar de entrada del líquido irrigante, reduciendo la presión dinámica y la distensión del área quirúrgica. Un calibre de asa de corte del 27-28 Fr. permite resecar en poco tiempo una buena cantidad de tejido. Además, su forma redondeada sigue la curva interna de la cápsula prostática.

TABLA I. PACIENTES: CARACTERÍSTICAS PREOPERATORIOS Y FECHA DE INTERVENCIÓN.

\begin{tabular}{|l|c|}
\hline Periodo de evaluación & $1985-2004$ \\
\hline No de Casos & 1017 \\
\hline Edad media & 68,9 \\
\hline Porcentaje de pacientes mayores de 70 años & 43 \\
\hline Pacientes con ASA establecido & 302 \\
\hline Riesgo Quirúrgico ASA-1 \% & 46 \\
\hline Riesgo Quirúrgico ASA-2 \% & 51 \\
\hline Riesgo Quirúrgico ASA-3 \% & 3 \\
\hline Tiempo medio de RTU en minutos ( $\mathrm{n}=310)$ & 65,1 \\
\hline Media en gramos de Tejido Resecado $(\mathrm{n}=840)$ & 32 \\
\hline Numero de Cirujanos (n $=894)$ & 5 \\
\hline
\end{tabular}




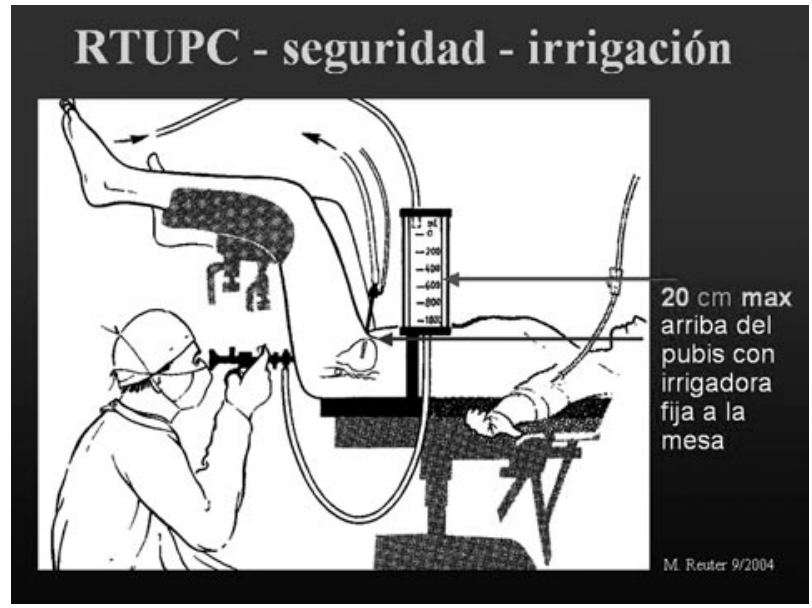

FIGURA 1. Esquema de la irrigación a baja presión y la situación del paciente y cirujano.

El resectoscopio se conecta a una unidad electroquirúrgica de corriente de alta frecuencia con ajuste automático según la resistencia eléctrica del tejido al corte (Figura 3). Su potencia máxima está limitada a 180 Watts para el corte y a 50 Watts para la coagulación. Esto procura un corte liso, uniforme, y preciso y una coagulación efectiva incluso de los senos venosos de las paredes.

El mango tipo pistola del resectoscopio permite un contacto muy sensible con la mano del cirujano y facilita el movimiento del asa de la corte incluso en áreas periféricas, por ejemplo, en la región latero apical de la próstata cerca del esfínter externo, la base lateral de la próstata o las vesículas seminales. El paño quirúrgico de recubrimiento debe permitir el tacto rectal frecuente. El tacto es vital para una resección dorsal segura y para la prevención de una contracción imprevista del músculo del suelo pélvico al levantar el suelo prostático.

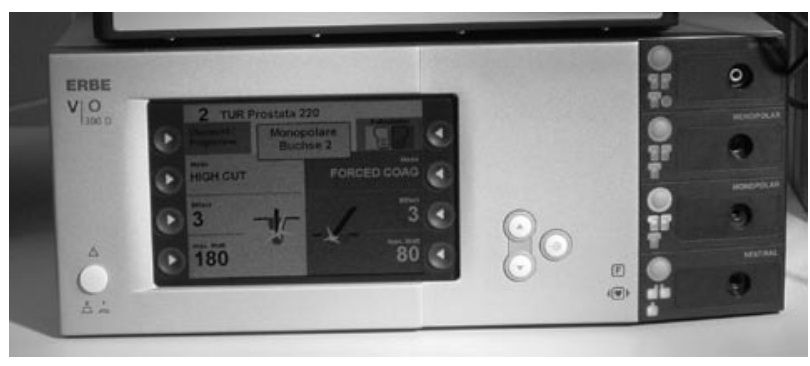

FIGURA 3. Unidad electroquirúrgica con corriente autorregulada de corte y coagulación.

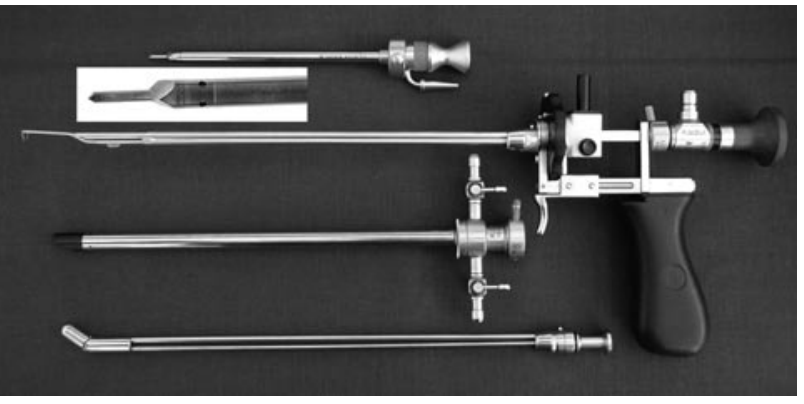

FIGURA 2. Resectoscopio del 28 Fr. con la clásica punta en pico de pato de McCarthy, trocar suprapúbico de Reuter.

El vídeo monitoreo de alta resolución magnifica el campo operatorio y consigue una diferenciación visual más fácil y fiable de los tejidos. Otra ventaja es que permite al cirujano situarse en una posición más relajada.

Las arterias se coagulan de forma inmediata a nivel de la cápsula así como las paredes de los senos venosos, para reducir su calibre y conseguir su cierre por trombosis local.

La absorción de fluidos la medimos con examen de expiración "Alcotest" añadiendo un 1\% de etanol al líquido de irrigación (6).

Comenzamos la intervención bajo anestesia con intubación con una linfadenectomía laparoscópica de las cadenas ilíacas externas y obturatriz para estadiaje (anestesia espinal en RTU sin linfadenectomía). En la misma sesión resecamos completamente la próstata y las vesículas seminales, entre el cuello vesical y la uretra, incluyendo el verumontanum y la cápsula prostática verdadera.

Para su estadiaje, las muestras se extraen por partes, en el orden siguiente: los tres lóbulos uno a uno, verumontanum, los dos cuadrantes dorsales de la cápsula verdadera (a las 6-9 y a las 3-6 del reloj endoscópico), las vesículas seminales, la porción terminal de los conductos deferentes, los pedículos prostáticos a las 5 y a las 7 (vasos y nervios prostáticos superiores) y, finalmente, los dos cuadrantes ventrales de la cápsula (Figura 7).

Al terminar ponemos un catéter suprapúbico del $14 \mathrm{Fr}$. a permanencia a través de la vaina semilunar del trocar y otro transuretral de tipo curvo 


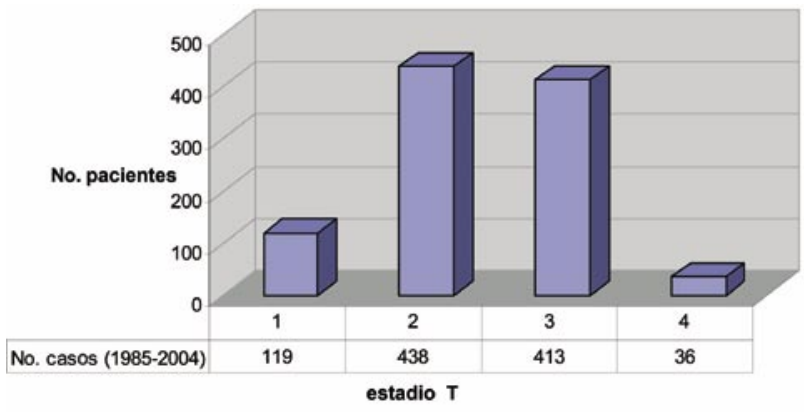

FIGURA 4a. Distribución según el estadio T postoperatorio.

antihematúrico con la ayuda de un introductor y del tacto rectal. Si fuera preciso, podemos controlar la situación del catéter a través de visión endoscópica por la vaina del trocar. Inflamos el balón con 40 c.c. y a lo largo de la mañana se mantiene una tracción suave de unos 250 grs. La irrigación se conecta al catéter suprapúbico y la altura del reservorio no debe exceder de 50 cents. sobre el pubis del paciente. Hacemos químio profilaxis con un antibacteriano de rutina (Bactrim en nuestro medio), comenzando con una dosis intraoperatoria, siguiendo con vía i.v. para continuar con medicación oral en los días siguientes. La profilaxis tromboembólica se lleva a cabo con heparina, medias compresoras y movilización precoz al día siguiente. La sonda transuretral la mantenemos durante cuatro días.

Al cuarto día postoperatorio se practica control de la fosa con un cistograma, eventualmente combinado con urografías. Cerramos el catéter suprapúbico al sexto día para iniciar el vaciado miccional y lo retiramos cuando el residuo postmiccional es nulo - menor de 50 c.c. En la mayoría de los casos el paciente es dado de alta al octavo día.

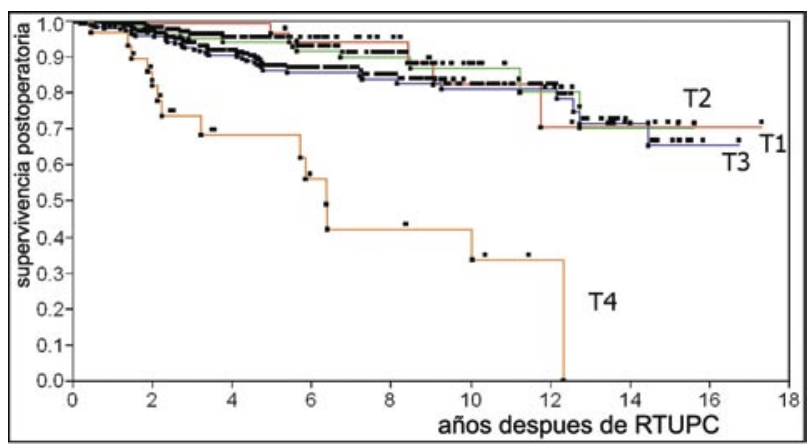

FIGURA 5. Supervivencia postoperatoria tras RTUPC radical, curva de Kaplan-Meier.

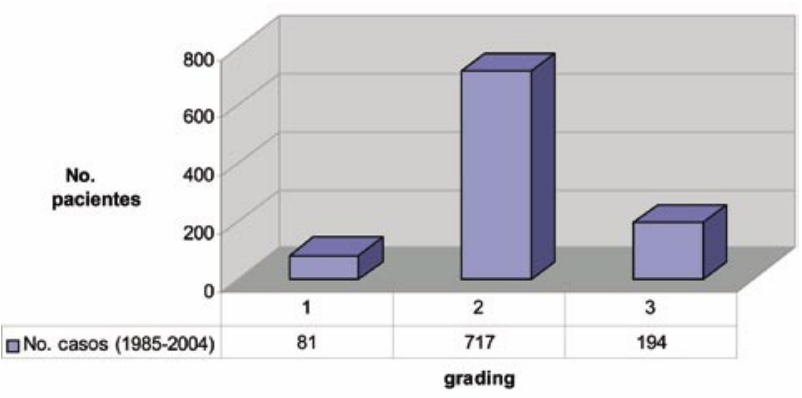

FIGURA 4b. Distribución según Grado postoperatorio.

Llevamos a cabo una segunda sesión a las 8-12 semanas para reducir el riesgo de márgenes positivos y restos de la cápsula prostática, similar a la "segunda sesión de seguridad" en el cáncer de vejiga. Resecamos nuevamente los pedículos vasculares y los cuadrantes de la fosa. En pacientes con potencia, la resección debe preservar las áreas nerviosas a las 5 y 7 del reloj. Los cuidados postoperatorios son similares a los de la primera intervención.

El seguimiento postoperatorio se realiza cada 3 meses mediante PSA sérico, tacto rectal y ecografía transrectal durante el primero y segundo años, después cada 6 meses en los cinco años posteriores, haremos gammagrafia ósea y TAC-scan. Más tarde se controlará cada año.

\section{RESULTADOS}

El estadio histopatológico determinado con el análisis del material quirúrgico se describe en la Tabla II (Figura 4a-b): T1 $\mathrm{n}=119, \mathrm{~T} 2 \mathrm{n}=438, \mathrm{~T} 3$

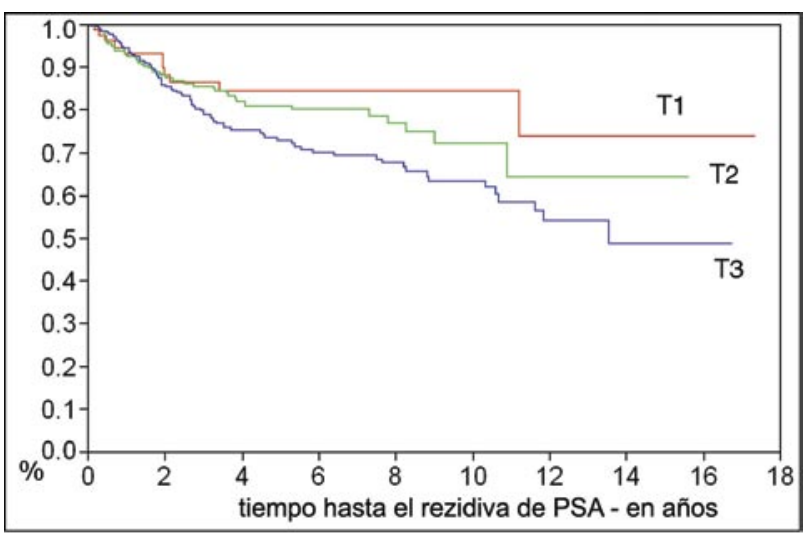

FIGURA 6. PSA-recidiva tras RTUPC radical, curva de Kaplan-Meier. 
TABLA II. ESTADIO HISTOPATOLÓGICO POSTOPERATORIO.

\begin{tabular}{|c|c|c|c|c|c|c|c|}
\hline Estadio & T1 & T2 & T3 & T4 & G1 & G2 & G3 \\
\hline No. casos (1985-2004) & 119 & 438 & 413 & 36 & 81 & 717 & 194 \\
\hline$\%$ & 11.8 & 43.5 & 41.1 & 3.6 & 8.2 & 72.3 & 19.6 \\
\hline
\end{tabular}

$\mathrm{n}=413, \mathrm{~T} 4 \mathrm{n}=36, \mathrm{G} 1 \mathrm{n}=81, \mathrm{G} 2 \mathrm{n}=717, \mathrm{G} 3$ $\mathrm{n}=194$ (el grado de Gleason no fue disponible en los 10 primeros años del estudio y por esto no son reflejados).

Las metástasis linfáticas incidentales fueron $\mathrm{pN} 1$ en 8 pacientes, pN2 en 5 pacientes, pNO en 690 pacientes, se practicó linfadenectomía en 703 pacientes.

En cuatro pacientes se apreció absorción de fluidos en cantidad menor de 50 c.c. en una serie consecutiva de 100 pacientes durante 2003-2004.

La vejiga se desplazó en sentido superior (en alrededor de un tercio de los pacientes) debido a la resección del tejido conectivo que rodea las vesículas seminales. Esto dificultaba la inserción final del catéter transuretral, por lo que en 4 casos hubo de ser corregida su posición mediante cistoscopia a través

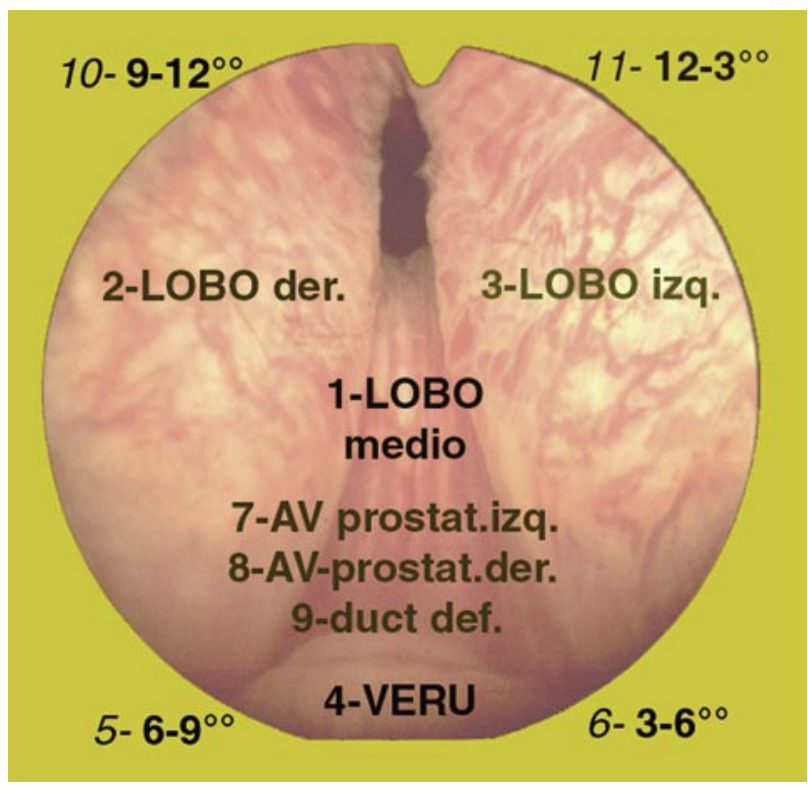

FIGURA 7. Esquema de la resección del tejido prostático siguiendo el horario endoscópico. de la vaina del trocar suprapúbico. La sonda hubo de ser introducida con la ayuda de la vaina semilunar accesoria del uretrotomo en dos casos. En ningún caso fue precisa la revisión quirúrgica.

Las complicaciones perioperatorias se detallan en la Tabla III: La transfusión sanguínea intra- y postoperatoria fue necesaria en el $4,4 \%$ de los casos ( $n=26$ de los 586 casos con datos disponibles). Revisión endoscópica por hemorragia fue precisa en el $2,5 \%$ de los casos (25 casos para $n=1017$ ). Nunca hubo necesidad de practicar cirugía abierta por complicaciones de la RTU.

Un paciente murió por embolia pulmonar masiva tras linfadenectomía abierta de estadiaje con RTU de seguridad (en las series anteriores a 1995). Una reimplantación ureteral y una revisión por hemorragia fueron necesaria por lesión accidental durante una linfadenectomía laparoscópica.

Las complicaciones postoperatorias tardías se detallan en la Tabla IV.

La estenosis del cuello vesical con necesidad de incisión ocurrió en el 8,9\% de los casos, la mayoría en el periodo posterior a 1995 debido a una resección más radical.

La incontinencia clasificada en tres grados por el ICS (International Continence Society) se manifestó tras un año de la RTUPC con una tasa del 13\% de grado 1 y del $2,5 \%$ de grado 2 . Ningún paciente presentó incontinencia grado 3 con pérdidas nocturnas.

La capacidad eréctil se conservó postoperatoriamente en el $39,2 \%$ de los pacientes con estadio T1-2 y en el $11 \%$ con T3 (sólo terapia antiandrogénica sin análogos $\mathrm{LH}$.). Estos pacientes no fueron seleccionados por la edad, potencia previa ni medicación.

La supervivencia postoperatoria (1985-2004) a 5/10 años fue en $\mathrm{T} 1: 97 / 82 \%$, en $\mathrm{T} 2: 95 / 87 \%$, en T3: $86 / 81 \%$ y en los pacientes T4: 68/34 \% (Figura 
5). (Estimación de Kaplan-Meier de la supervivencia separada por estadios T1 al T4.)

En el seguimiento postoperatorio se consideró recurrencia bioquímica un aumento de los valores de PSA en tres determinaciones consecutivas teniendo en cuenta el estadio-T. A los 5 años la PSA-recidiva fue del $15 \%$ en los $\mathrm{T} 1,19 \%$ en los $\mathrm{T} 2$ y $27 \%$ en los T3. Lo reflejamos en la Figura 6 (Estimación de Kaplan-Meier de la Recidiva Bioquímica según Estadios T 1-3.) (Tabla VI).

\section{DISCUSIÓN}

Si la RTU es el "estándar de oro" para el adenoma de próstata, es una consecuencia lógica el que esta se quiera extender más allá de la cápsula para una resección completa de la próstata cuando existe cáncer. Esto ya se intentó hace 70 años, con resultados inaceptables debido a las deficiencias del equipo y a la propia ejecución del procedimiento. Además no se tuvieron en cuenta los aspectos oncológicos. Durante un largo proceso, he-

TABLA III. COMPLICACIONES PERIOPERATORIAS MAYORES Y MENORES DE LA RTUPC.

\begin{tabular}{|c|c|c|}
\hline \multicolumn{3}{|c|}{ Complicaciones Perioperatorias Mayores de la RTUPC } \\
\hline & Casos $/ n^{\circ}$ de pacientes & $\%$ \\
\hline Tasa de Transfusiones & $26 / 586$ & 4,4 \\
\hline Revisiones por Hemorragia & $25 / 1017$ & 2,46 \\
\hline Absorción de Fluidos. máx. de 50 c.c. & $4 / 100$ & 4,0 \\
\hline Embolismo Pulmonar & $3 / 1017$ & 0,29 \\
\hline Conversión a cirugía abierta & $0 / 1017$ & 0 \\
\hline Linfocele Sintomático & $4 / 1017$ & 0,39 \\
\hline Trombosis Periférica & $4 / 1017$ & 0,39 \\
\hline Perforación Peritoneal & $0 / 1017$ & 0 \\
\hline Obstrucción Ureteral & $0 / 1017$ & 0 \\
\hline Perforación Rectal Cubierta & $1 / 1017$ & 0,098 \\
\hline Hematoma Perivesical & $5 / 1017$ & 0,49 \\
\hline Mortalidad (linfadenectomía) & $1 / 1017$ & 0,1 \\
\hline \multicolumn{3}{|c|}{ Complicaciones Perioperatorias Menores } \\
\hline & No. & $\%$ de Casos \\
\hline Hemorragia retrasada por anticoagulación & $126 / 1017$ & 12,36 \\
\hline Dolor perineal & $136 / 1017$ & 13,33 \\
\hline Irritación peritoneal (dolorosa) & $26 / 1017$ & 2,55 \\
\hline Irritación periostal (dolorosa) & $2 / 1017$ & 0,2 \\
\hline Linfocele asintomático & $43 / 1017$ & 4,22 \\
\hline
\end{tabular}


TABLA IV. COMPLICACIONES POSTOPERATORIAS TARDÍAS.

\begin{tabular}{|l|l|l|}
\hline & No. & \% de Casos \\
\hline Incisión por Esclerosis de Cuello Vesical (n=1017) & 91 & 8,9 \\
\hline Incontinencia (1 año postop.) grado $1(\mathrm{n}=560)$ & 73 & 13 \\
\hline Incontinencia (1 año postop.) grado 2(n=560) & 14 & 2,5 \\
\hline Incontinencia (1 año postop.) grado 3(n=560) & 0 & 0 \\
\hline Preservación de la Erección en est. T1-2 (n=283) & 107 & 37,8 \\
\hline Preservación de la Erección en est. T3a $(\mathrm{n}=246)$ & 27 & 11 \\
\hline Diseminación metastásica postoperatoria $(\mathrm{n}=1017)$ & 0 & 0 \\
\hline
\end{tabular}

mos devuelto el procedimiento a su fase actual. La innovación más importante fue la irrigación a baja presión con el nivel del líquido irrigante a menos de $20 \mathrm{~cm}$., preferiblemente a $10 \mathrm{~cm}$. por encima de la región púbica. La solución irrigante ha sido estandarizada, incorporamos la unidad electroquirúrgica auto-regulada y la imagen endoscópica esta ahora video-monitorizada y magnificada en una pantalla de alta resolución.

La irrigación es necesaria para aclarar el campo quirúrgico, y ya que el corte se realiza por corriente eléctrica, el líquido irrigante debe estar libre de electrolitos. Además su flujo ejerce una presión dinámica sobre el tejido y los senos venosos, y el relleno-llenado- de la vejiga aumenta la presión estática sobre la fosa. El aumento de la presión dinámica y estática aumenta el riesgo de absorción del fluido, que debe ser evitada para prevenir el síndrome-RTU y la diseminación de células cancerosas prostáticas. Esta prevención es mucho más efectiva con la baja presión.

Se ha comprobado en estudios in vitro e in vivo que un nivel de menos de $20 \mathrm{~cm}$. sobre el pubis es suficiente para prevenir la absorción y también

\section{TABLA V. DATOS ESTADÍSTICOS DE LA FIG.5: SUPERVIVENCIA SEGÚN EL ESTADIO T 1-4.}

\begin{tabular}{|c|c|c|c|c|c|c|}
\hline \multicolumn{7}{|c|}{ Conjunto de Supervivencia según el Estadio T. } \\
\hline Grupo & № Fallecidos & \multicolumn{3}{|c|}{ № Censados } & Media & Std. Error \\
\hline 1 & 6 & 113 & \multicolumn{2}{|l|}{10.963} & Parcial & 0.37148 \\
\hline 2 & 19 & 411 & \multicolumn{2}{|l|}{115.916} & Parcial & 0.28354 \\
\hline 3 & 41 & 356 & \multicolumn{2}{|l|}{123.186} & Parcial & 0.31608 \\
\hline 4 & 14 & 21 & \multicolumn{2}{|l|}{719.945} & & 0.99239 \\
\hline Agrupados & 80 & \multicolumn{3}{|c|}{122.855} & Parcial & 0.23004 \\
\hline \multicolumn{7}{|c|}{ Prueba Entre Grupos } \\
\hline Test & ChiSquare & \multicolumn{2}{|c|}{ DF } & \multicolumn{3}{|l|}{ Prob $>$ ChiSq } \\
\hline Log-RanK & 454.596 & \multicolumn{2}{|c|}{3} & \multicolumn{3}{|l|}{$<.0001$} \\
\hline Wilcoxon & 279.810 & \multicolumn{2}{|r|}{3} & \multicolumn{3}{|c|}{$<.0001$} \\
\hline
\end{tabular}




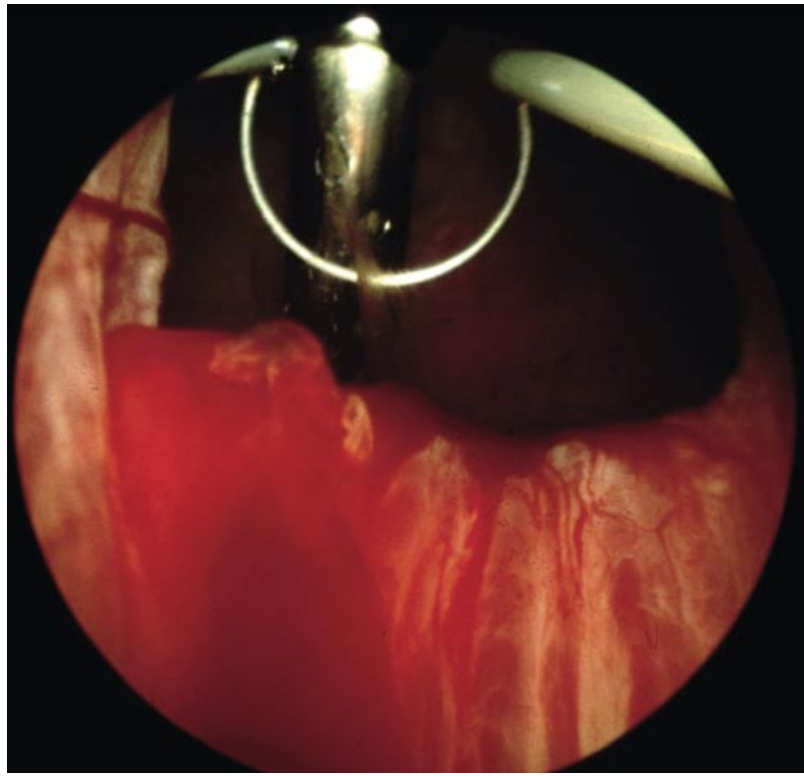

FIGURA 7A. Visión distal. Visión del asa y el trocar.

suficiente para limpiar la fosa de sangre y tejido resecado, siendo un efecto secundario favorable el que los pequeños sangrados se vean mejor y se coagulen más fácilmente. El flujo laminar con una vaina de resectoscopio del $28 \mathrm{Ch}$. mejora la visión, mientras que con una del $24 \mathrm{Ch}$. se producen turbulencias y obscurece la misma.

Hoy día, la solución irrigante es un producto farmacéutico, semiosmolar, libre de electrolitos, esté-

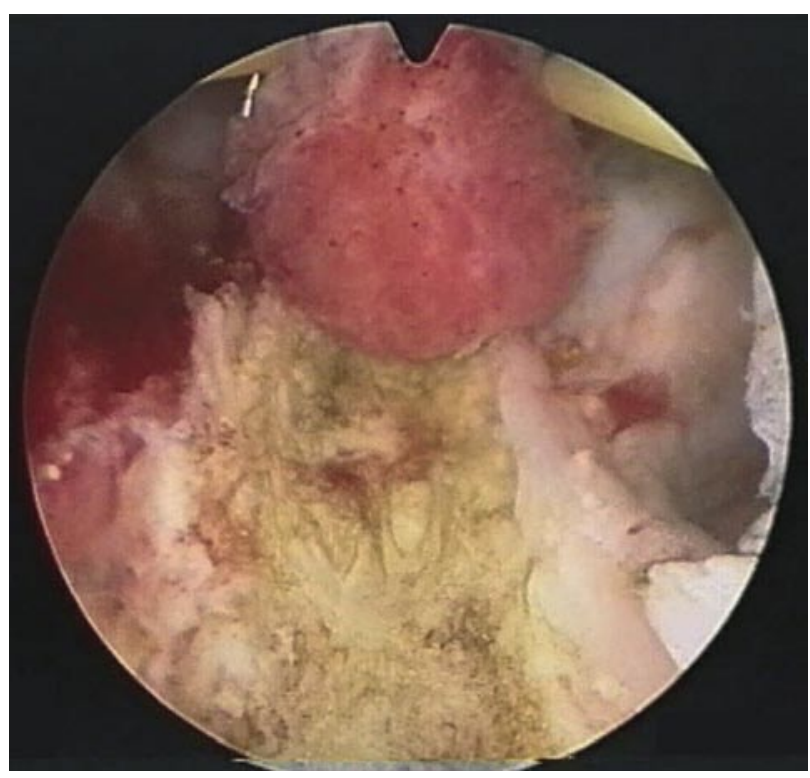

FIGURA 7B. Ductus eyaculatorii después de la resección del veru a tejido en el asa de resección.

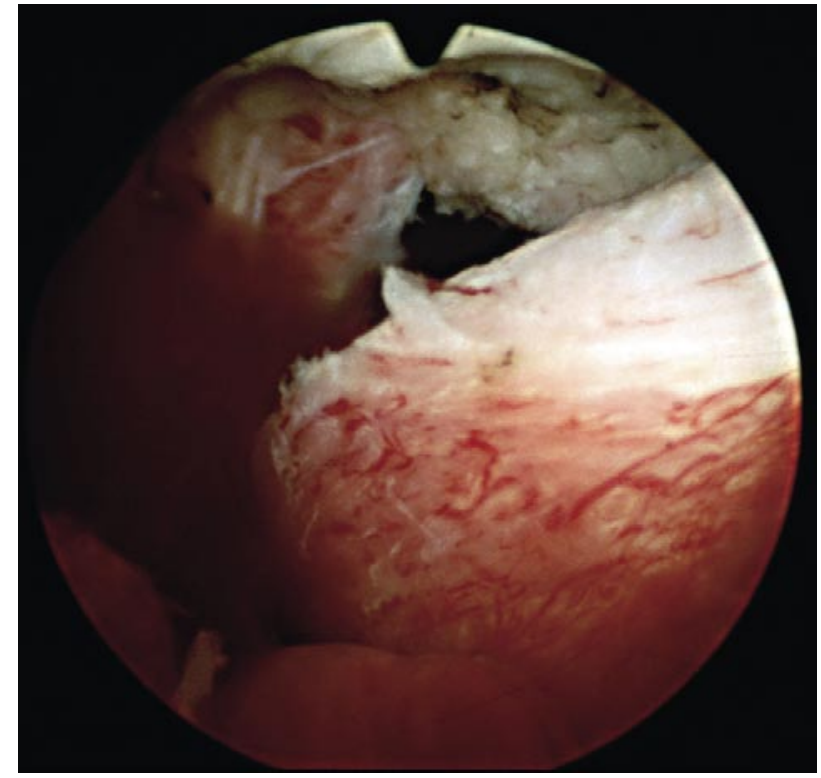

FIGURA 7A-2. Resección del lóbulo izquierdo.

ril y libre de pirógenos. Aumentando así la seguridad del procedimiento.

La unidad electroquirúrgica produce una corriente para corte y coagulación que es autorregulada mediante feedback automático según la resistencia del tejido. Esto reduce la corriente aplicada al mínimo necesario y, como consecuencia, la chispa de corte es casi invisible, siendo el efecto de corte casi sin necrosis y evitando prácticamente el daño a

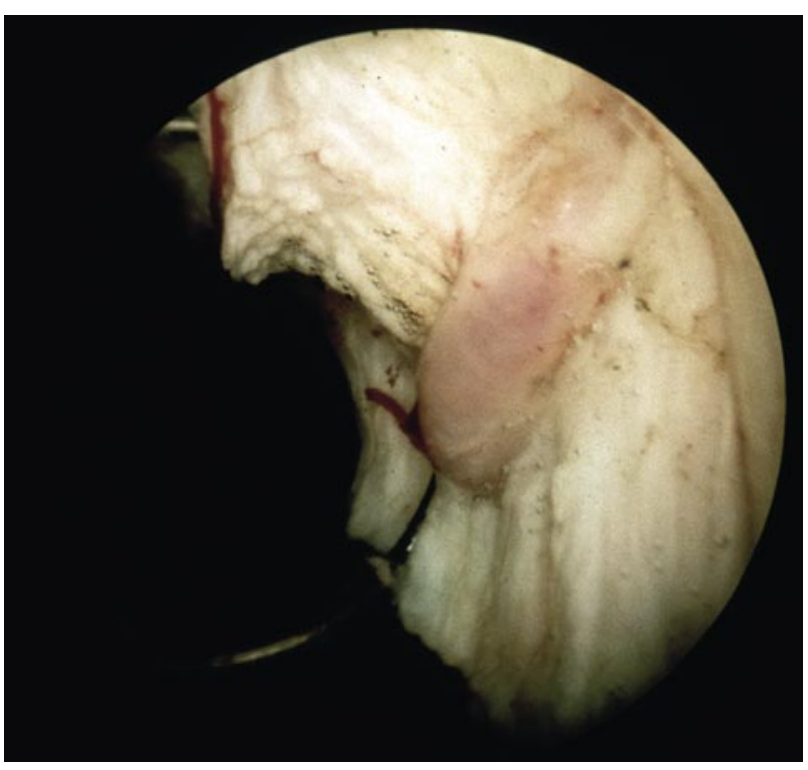

FIGURA 7C. Arteria periprostática sin daño. 


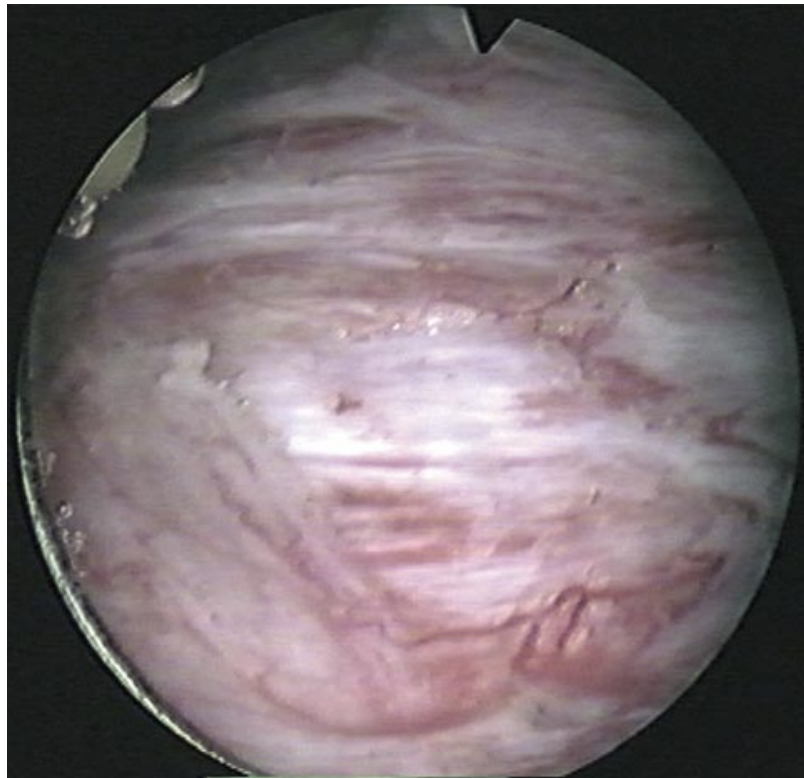

FIGURA 7D. Fascia Denonvilliers.

los nervios esfinterianos y erectores. Las paredes de los vasos sanguíneos son fácilmente conservadas y la coagulación de las paredes de los senos venosos reduce su apertura, facilitando su cierre por trombosis local.

El video-monitoreo con alta resolución (SVHS) aumenta la imagen alrededor de 30 veces, facilitando de este modo la diferenciación de los tejidos - prostáticos/ periprostáticos/ músculo del cuello vesical/ pared de la uretra membranosa/ apertura

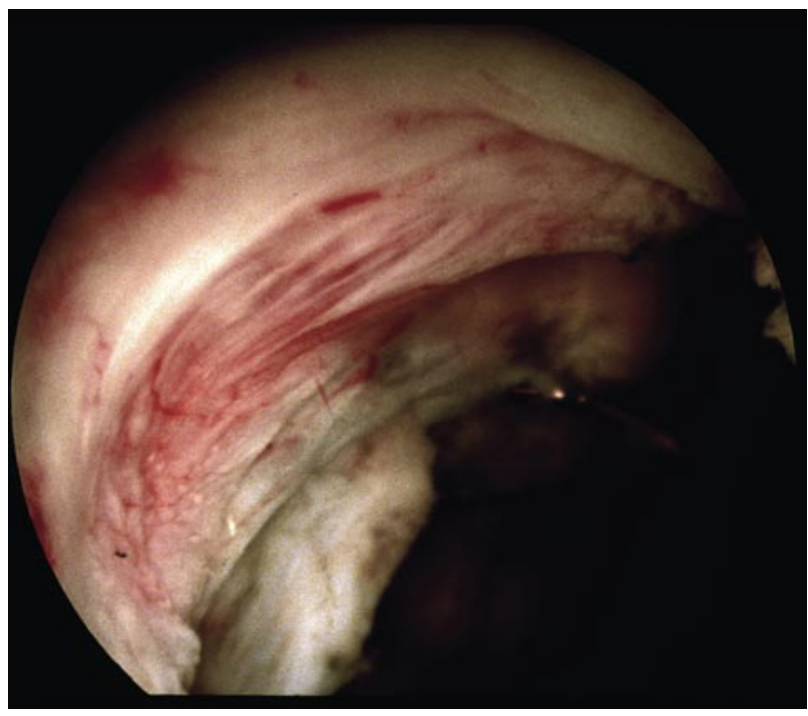

FIGURA 7E. Músculo elevador del ano. de las vesículas seminales - y permite al cirujano mantener una postura relajada.

Los primeros protocolos de la RTUP fueron establecidos en 1940 por urólogos alemanes, y se aplican aún frecuentemente para la RTUP del adenoma (7): El tiempo de RTU se limita a una hora y el peso hasta 40 - $50 \mathrm{grs}$. El verumontanum no se reseca y la perforación capsular es una temida complicación. Las vesículas seminales están fuera del alcance de la RTU. La norma era limitar el riesgo de RTU-síndrome y de la incontinencia a unos niveles tolerables con la RTU convencional.

Sin embargo, la RTU del cáncer de próstata no es posible siguiendo estas reglas porque, ante todo, la cápsula debe ser perforada para una completa resección. En grandes próstatas una hora no es tiempo suficiente, y el verumontanum es parte del tejido prostático.

La clave para obviar estas normas es la irrigación a baja presión. De este modo la cápsula puede ser resecada sin límite de tiempo, previene la absorción de fluido y la pérdida de sangre se reduce con un mejor y más espontáneo control del sangrado arterial y venoso. El verumontanum puede ser resecado, porque es justamente la línea marcadora, y su ausencia no influye sobre la continencia urinaria, hecho conocido a través de la prostatectomía radical.

Se observan las normas oncológicas, ya que la intervención comienza con la disección linfática laparoscópica para el estadiaje, la próstata se reseca de forma completa (Figura 7: esquema de la toma de tejido), incluyendo la totalidad de la cápsula y el tejido periprostático. Se toman biopsias de los márgenes y se resecan completamente el verumontano y las vesículas seminales (Figuras 7B,G). Cuando se reseca la cápsula prostática quedan expuestos los conductos eyaculadores y la porción terminal de las vesículas seminales desde donde pueden ser resecadas completamente (Figuras 7G,H). Los límites de la cápsula prostática, con la pared vesical encima y la uretra membranosa debajo, pueden ser identificados con ayuda de la magnificación x 30 de la imagen de video (Figuras 7 C-E, L-N). Si cualquier duda surge en cuanto a los márgenes, una biopsia sometida a una sección congelada la despejará. La extensión perineural del cáncer ocurre frecuentemente, por lo que se toma una biopsia separada desde el pedículo superior con los vasos prostáticos superiores y nervios autónomos (Figuras $7 \mathrm{I}, \mathrm{K}$ ) Se previene la diseminación con la baja presión. El oncólogo puede tener reticencias por la fragmentación necesaria cuando se reseca transuretralmente. Pero, por otra parte, durante la cirugía abierta y laparoscópica la glándula es 
exprimida y desgarrada para obtener acceso a todos los lugares; siendo el riesgo de diseminación tumoral similar en ambos procedimientos. Otra ventaja de la RTUPC es que disminuye el riesgo de márgenes positivos con una segunda intervención de seguridad tras 8-12 semanas, en la que el tejido residual es resecado de nuevo con nueva toma de biopsias. Mas adelante, posteriores RTUs pueden practicarse por recidivas locales, normalmente con buen resultado.

El reseccionista debería estar capacitado para resecar con baja presión de irrigación unos 80 grs. de tejido adenomatoso (aprox. 110 c.c. de volumen) en menos de 80 minutos, con un riesgo menor

TABLA VI. DATOS ESTADÍSTICOS DE LA FIG. 6: RECIDIVA BIOQUIIMICA SEPARADA POR ESTADIOS T A LOS 3, 5 Y 10 AÑOS.

\begin{tabular}{|c|c|c|c|c|}
\hline \multicolumn{5}{|c|}{ Supervivencia Libre de PSA según el Estadio T. } \\
\hline Tiempo de PSA-rec.-años & $\%$ PSA-libre & \%Fracasos & Superv.StdErr & №en Riesgo \\
\hline \multicolumn{5}{|l|}{ Estadio T 1} \\
\hline 0.0000 & 1,00 & 0.0000 & 0.0000 & 74,00 \\
\hline 0.9582 & 0.9296 & 0.0704 & 0.0304 & 64,00 \\
\hline 2.9651 & 0.8638 & 0.1362 & 0.0425 & 44,00 \\
\hline 4.9774 & 0.8432 & 0.1568 & 0.0462 & 35,00 \\
\hline 9.9986 & 0.8432 & 0.1568 & 0.0462 & 9,00 \\
\hline \multicolumn{5}{|l|}{ Estadio T 2} \\
\hline 0.0000 & 1,00 & 0.0000 & 0.0000 & 290,00 \\
\hline 1.0021 & 0.9296 & 0.0704 & 0.0152 & 257,00 \\
\hline 2.9952 & 0.8559 & 0.1441 & 0.0219 & 159,00 \\
\hline 4.9938 & 0.8093 & 0.1907 & 0.0269 & 94,00 \\
\hline 10.0205 & 0.7210 & 0.2790 & 0.0464 & 19,00 \\
\hline \multicolumn{5}{|l|}{ Estadio T 3} \\
\hline 0.0000 & 1,00 & 0.0000 & 0.0000 & 266,00 \\
\hline 1.0157 & 0.9424 & 0.0576 & 0.0144 & 242,00 \\
\hline 3.0089 & 0.7928 & 0.2072 & 0.0263 & 166,00 \\
\hline 4.9993 & 0.7274 & 0.2726 & 0.0302 & 110,00 \\
\hline 10.0315 & 0.6329 & 0.3671 & 0.0378 & 47,00 \\
\hline \multicolumn{5}{|l|}{ Tests entre Grupos T } \\
\hline Test & are & DF & Prob>ChiSq & \\
\hline Log-Rank & & 3 & $<.0001$ & \\
\hline Wilcoxon & & 3 & $<.0001$ & \\
\hline
\end{tabular}




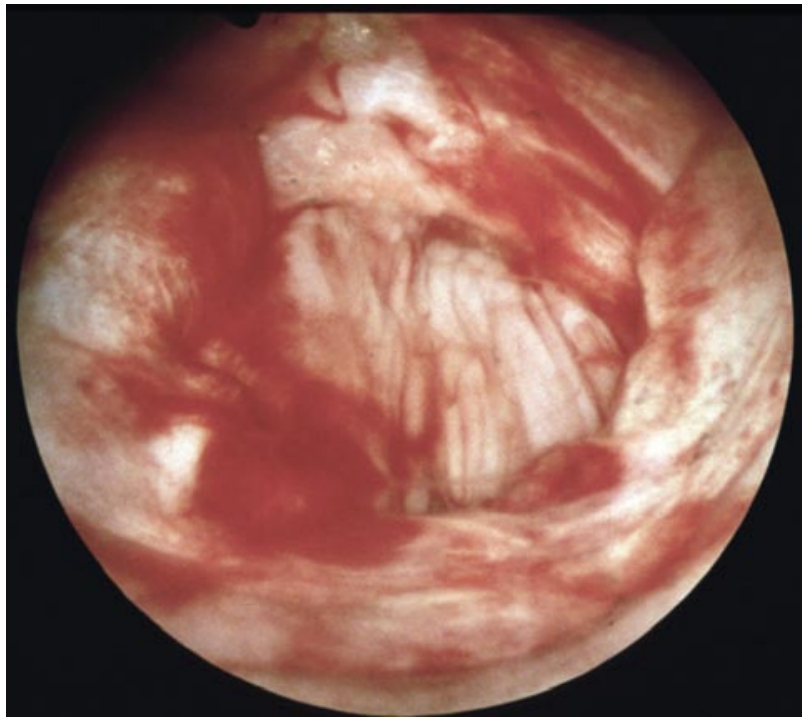

FIGURA 7F. Músculo del recto.

del $10 \%$ de transfundir sangre. Las medidas de seguridad descritas anteriormente y desarrolladas en un largo periodo de décadas deben observarse meticulosamente. El instrumental y la unidad electroquirúrgica descritos han hecho de este un procedimiento seguro.

Para el mismo, son subsidiarios aquellos pacientes con cáncer de próstata localizado, cuando la cirugía abierta no es una opción por el riesgo operatorio, edad, condicionantes religiosos, obesidad o

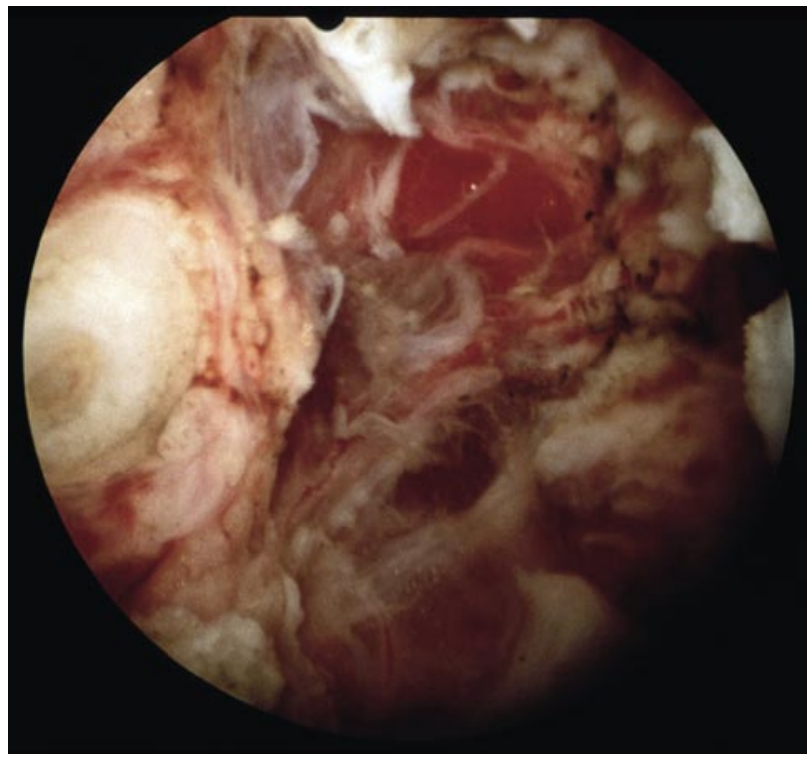

FIGURA 7H. Fosa retrovesical tras resección completa de las vesículas seminales, conducto deferente izquierdo seccionado.

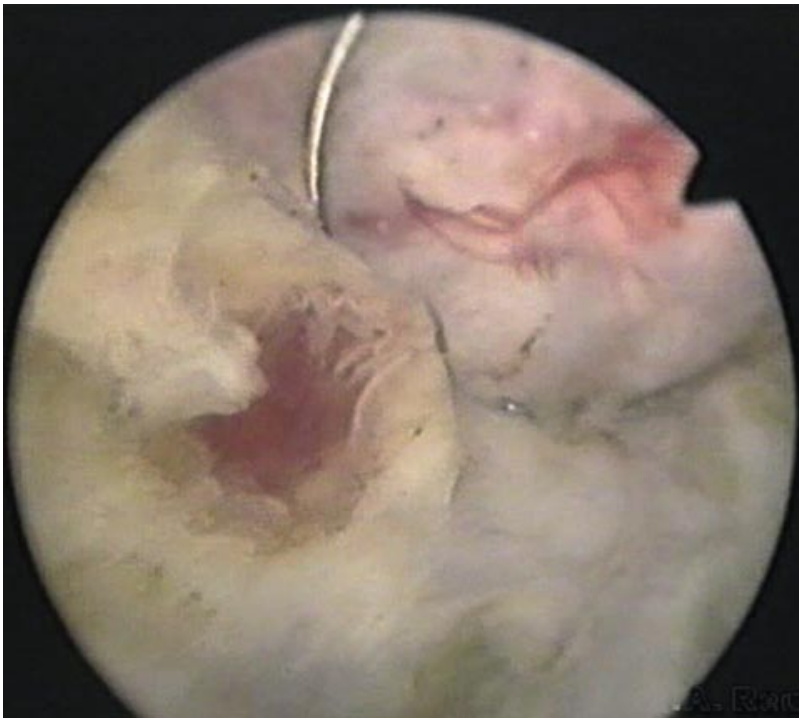

FIGURA 7G. Aspecto tras la resección de la vesícula seminal.

tratamientos previos con radioterapia o braquiterapia. Las recidivas locales pueden ser resecadas en posteriores sesiones como una opción previa a radioterapia o tratamiento antiandrógeno.

Las complicaciones que pueden ocurrir son básicamente las mismas que en la cirugía radical. La lesión del recto es posible y, de hecho, ocurre (las fibras musculares longitudinales del recto se ven expuestas, Figura 7F). Sin embargo es raro que se produzca una perforación gracias al corte delicado de

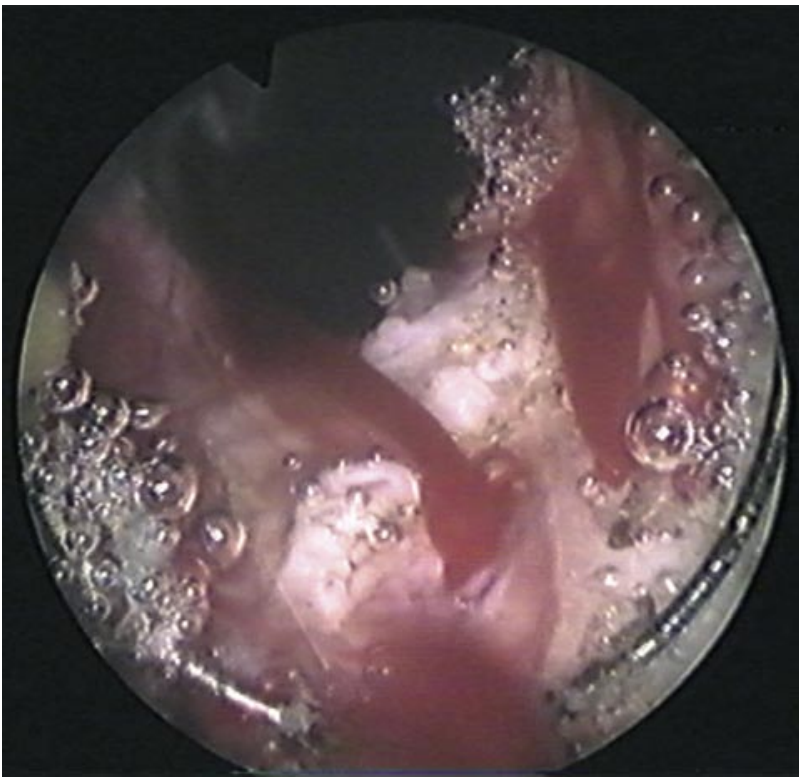

FIGURA 7I. Pedículo superior con los vasos prostáticos superiores izquierdos tras la toma de una biopsia separada - sangrantes. 


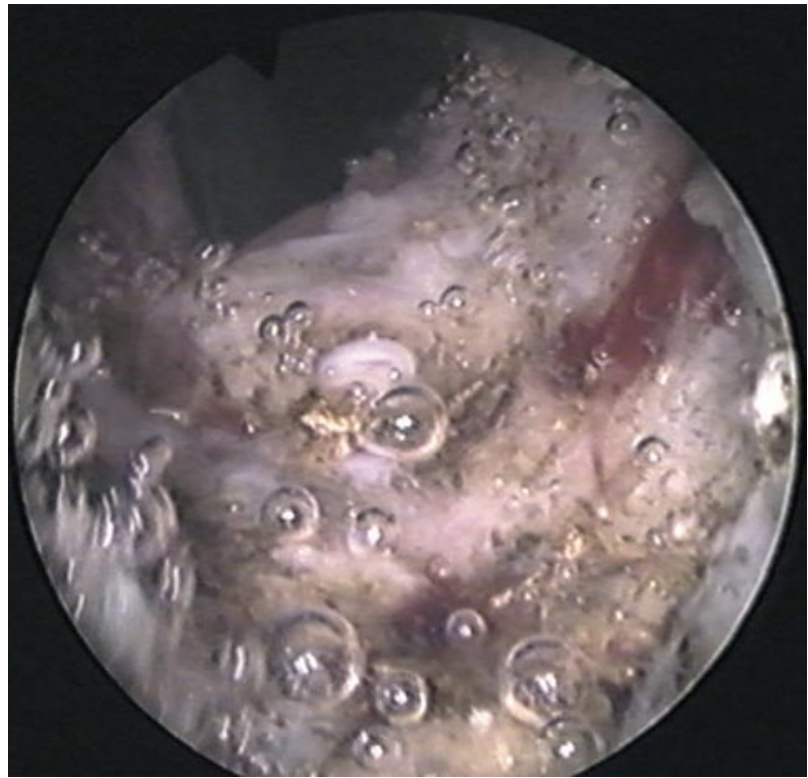

FIGURA 7K. Pedículo superior tras la coagulación.

la unidad electroquirúrgica y a la magnificación del campo.

La lesión del uréter nunca ocurre durante la RTU ya que su orificio se identifica normalmente en el trígono. La resección superficial del orificio ureteral se da si existe obstrucción y podemos asegurarnos colocando un stent, aunque esto es necesario sólo si

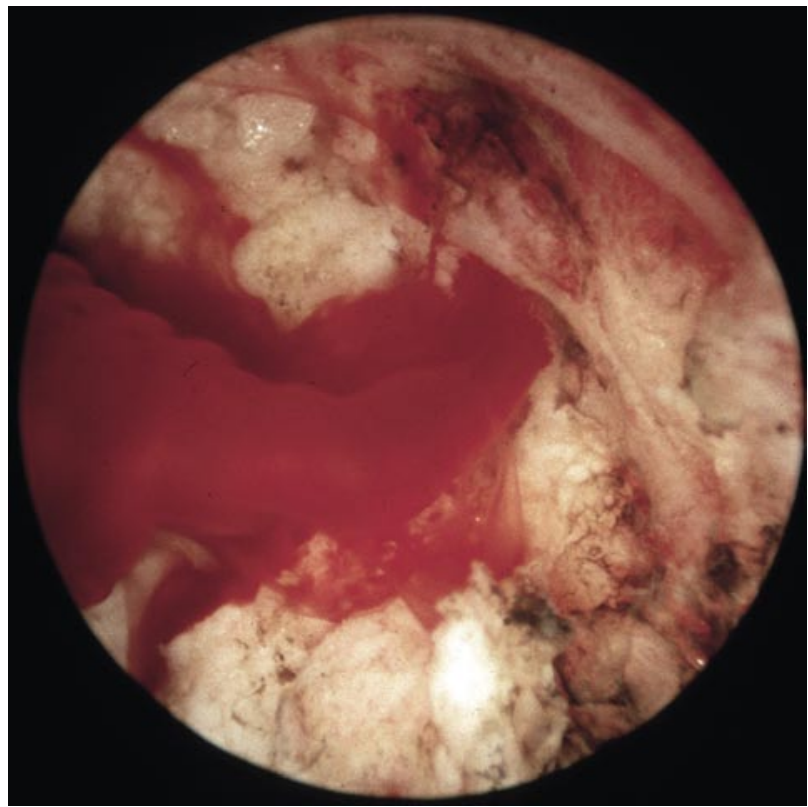

FIGURA 7M. Seno venoso del plexo Santorini abierto sangrante.

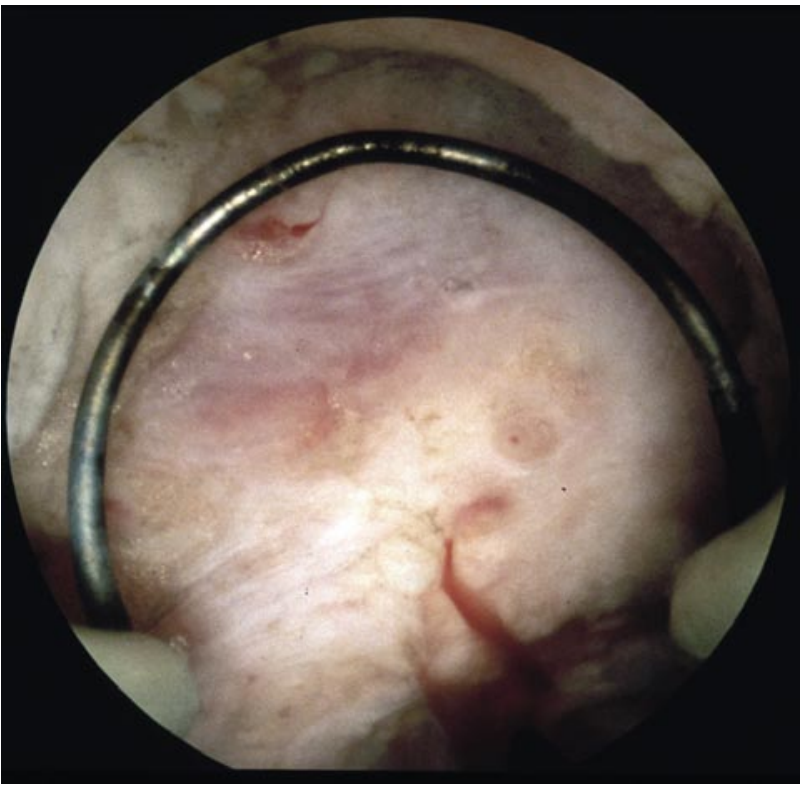

FIGURA 7L. Seno venoso del plexo Santorini.

esta infiltrado por el tumor. El riesgo de lesión ureteral es mínimo cuando se resecan las vesículas seminales porque esta bien protegido por tejido conectivo (Figura 7H). La perforación peritoneal ocurre solamente si ha sido infiltrado por el tumor en un caso incurable. La media de transfusión sanguínea intra y postoperatoria es del $4,4 \%$, por lo que hemos dejado de practicar la auto-transfusión.

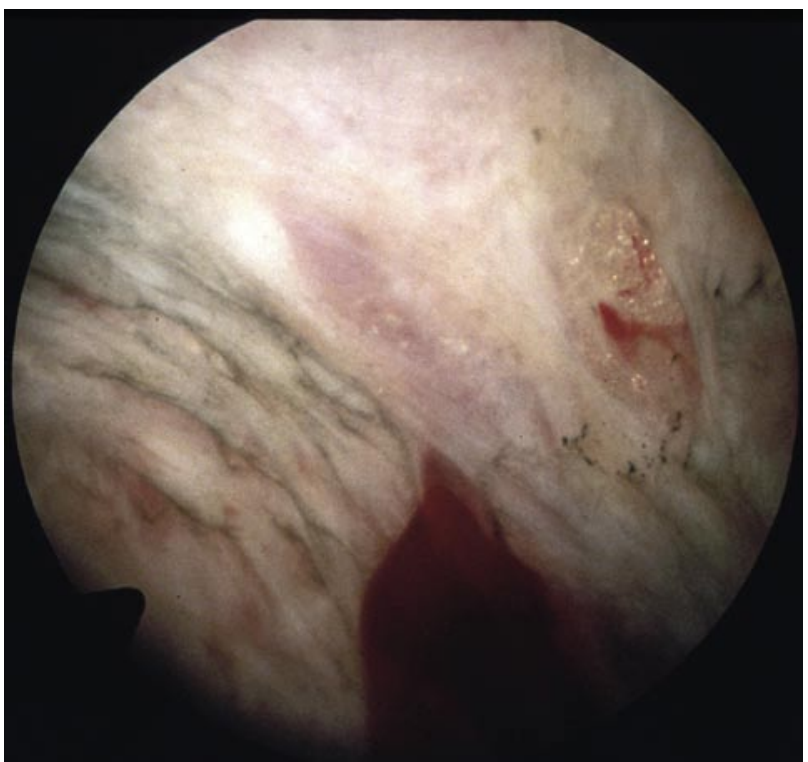

FIGURA 7N. Tejido periprostático anterior con seno sangrante. 
La incontinencia suele ser debida a la existencia previa de una insuficiencia del suelo pélvico y/o causada por lesión nerviosa. La lesión directa del músculo esfinteriano no debe ocurrir en una cirugía cuidadosa.

Aunque la edad media en RTUPC es mayor que en la cirugía abierta, es bajo el índice de incontinencia: 2,5\% para el grado 2, (pacientes sin incontinencia nocturna). El riesgo de lesión del nervio cavernoso de la erección depende de la situación de dicho nervio respecto al tumor y a la cápsula. El riesgo de disfunción eréctil en nuestra serie en pacientes no seleccionados con estadio $\mathrm{T} 1-2$ es del $62 \%$. Sin embargo, debemos considerar la elevada edad media de los pacientes, con mas de 70 años en nuestras series. Otros factores son causa de impotencia comparados con la intervención, siendo mayor su prevalencia en pacientes jóvenes. Estas funciones subjetivas son normalmente investigadas en un estudio de calidad de vida, que ya está en marcha.

La complicación mas frecuente es la estenosis del cuello vesical, que puede ser tratada tempranamente con dilataciones hasta el 16 Fr. La incisión fría y opcionalmente la vaporización con láser de la cicatriz fue necesaria en el $8,9 \%$ de los casos. El riesgo de esclerosis de la fosa ha aumentado significativamente con la radicalidad de la técnica desde 1995. Hay pocas complicaciones graves, como perforación transrectal (menos 1 por mil), embolismo pulmonar $(0,39 \%)$. La mortalidad postoperatoria fue de 1 por 1017.

La media de supervivencia a los 10 años depende del estadio, siendo del $82 \%$ en los $\mathrm{T} 1,87 \%$ en los T2 y $81 \%$ en los pacientes con T3 (ver Figura 5). La recurrencia -recidiva- del PSA de los casos T1 y 2 es del $15 \%$ y $19 \%$ respectivamente y del $27 \%$ para los T3 a lo largo de 5 años (ver Figura 6). Estas cifras son equivalentes a los resultados publicados para la cirugía radical abierta y laparoscópica $(8,9,10)$.

\section{CONCLUSIONES}

Encontramos nuestra serie de 1017 casos de TURPC-radical (resección transuretral completa de la próstata y vesículas seminales por cáncer), después de 20 años, un procedimiento válido para pacientes no subsidiarios de cirugía abierta, dando unos resultados equivalentes de supervivencia y recidiva, con baja tasa de complicaciones sobre todo de la incontinencia. La clave está en la irrigación a baja presión, equivalente a un nivel del líquido de irrigación a una altura de $10 \mathrm{~cm}$., junto con otros hechos importantes descritos previamente. Igual que en otros procedimientos oncológicos, se requiere una gran habilidad y entrenamiento con el endoscopio.

\section{BIBLIOGRAFIA y LECTURAS RECOMENDADAS ( ${ }^{*}$ lectura de interés $y^{* *}$ lectura fundamental)}

1. REUTER, H.J.: "Atlas of urologic endoscopic surgery”. Philadelphia, Saunders, p. 9-33, 1982. REUTER, H.J.: "Atlas der urologischen Endoskopie”. Stuttgart, New York, Thieme, p. 9-33, 1980.

2. MATOUSCHEK, E.: "Urologic endoscopic surgery". Toronto, p. 108-113, 1987.

MATOUSCHEK, E.: "Urologisch-endoskopische operationen". Stuttgart, Schattauer, p. 108-112, 1989.

3. VERGER-KUHNKE, A.; REUTER, M.A.; EPPLE, W. y cols.: "La resección transuretral de la próstata de baja presión hidráulica, resultados en 340 pacientes con adenomas grandes". Actas Urol. Esp., 30: 896, 2006.

4. REUTER, M.A.; REUTER, H.J.: "Prevention of irrigant absorption during TURP". Int. Urol. Nephrol., 10: 293, 1978.

**5. REUTER, M.A.; REUTER, H.J.; EPPLE, W.: "Electro resección transuretral total del carcinoma de la próstata". Arch. Esp. Urol., 44: 611, 1991.

6. HAHN, R.G.; OLSSON, J.: "Ethanol monitoring of the transurethral resection syndrome". J. Clin. Anesth., 8: 652, 1996.

7. REUTER, M.A.; REUTER, H.J.: "History of transurethral Prostatectomy in Europe". Reuter MA, Reuter HJ, Engel R.: History of Endoscopy, Stuttgart, Max-Nitze-Museum, pp 116-222, 2003.

8. HAN, M.; PARTIN, A.W.; PIANTADOSI, S. y cols.: "Era specific biochemical recurrence-free survival following radical prostatectomy for clinically localized prostate cancer". J. Urol., 166: 416, 2001.

*9. ROEHL, K.A.; HAN, M.; RAMOS, C.G. y cols.: "Cancer progression and survival rates following anatomical radical retropubic prostatectomy in 3,478 consecutive patients: long term results". J. Urol., 172: 910, 2004.

10. TRABLUSI, E.J.; GUILLONNEAU, B.: "Laparoscopic radical prostatectomy". J. Urol., 173: 1072, 2005. 